\title{
POSIBILIDADES DE LA WEB 2.0 EN ORIENTACIÓN EDUCATIVA: UN ESTUDIO EXPLORATORIO SOBRE SU PRESENCIA EN LAS WEB DE LOS DEPARTAMENTOS DE ORIENTACIÓN DE SECUNDARIA
}

\section{AFFORDANCES OF WEB 2.0 IN EDUCATIONAL GUIDANCE: AN EXPLORATORY STUDY ON THE WEB PRESENCE IN SECONDARY SCHOOLS GUIDANCE DEPARTMENTS}

\author{
Pablo-César Muñoz-Carril ${ }^{1}$ \\ Universidad de Santiago de Compostela. Facultad de Formación del Profesorado. \\ Lugo, España \\ Mercedes González-Sanmamed \\ Universidad de A Coruña. Facultad de Ciencias de la Educación. \\ A Coruña. España
}

\section{RESUMEN}

El esfuerzo en cuanto a la dotación de recursos TIC en las instituciones de secundaria ha sido considerable en los últimos años. Sin embargo, las investigaciones, a nivel nacional e internacional, indican que su uso es todavía limitado en los diversos procesos educativos que acontecen en las aulas y los centros. Concretamente, en el ámbito de la orientación educativa, a pesar de la utilidad que diversos autores adjudican a las herramientas TIC, los estudios desvelan que no se está aprovechando todo su potencial y que su empleo es todavía escaso.

Tomando como base esta situación, se ha diseñado un estudio para analizar la presencia de las herramientas de la denominada web 2.0 en orientación educativa. Se realizó una investigación no experimental y cuantitativa en la que revisamos las páginas de web de los 155 centros de educación secundaria de la provincia de la Coruña.

${ }^{1}$ Correspondencia: Pablo César Muñoz Carril. Facultad de Formación del Profesorado. Universidad de Santiago de Compostela. Departamento de Didáctica y Organización Escolar. Campus Terra. Avda. Ramón Ferreiro, s/n, CP 27002, Lugo (España). Correo-e: pablocesar.munoz@usc.es, web: http://www.equipoeira.net 
Los resultados muestran que más de la mitad de las webs analizadas poseen un entorno claro y amigable que permite la facilidad de uso por parte del usuario. Pero la disponibilidad de aplicaciones web 2.0 en las web de los Departamentos de Orientación es muy reducida, destacando fundamentalmente el empleo del correo electrónico y los blogs. Para facilitar una adecuada integración de las TIC sería necesario mejorar la capacitación tecnológica de los orientadores y ofrecerles apoyos e incentivos para que incorporen estas herramientas en su actividad profesional.

Palabras clave: herramientas web 2.0, departamentos de orientación, educación secundaria, orientación educativa, tecnologías de la información y de la comunicación.

\section{ABSTRACT}

Efforts to provide ICT resources to Secondary Schools have been significant in the recent years. However, research at both national and international level shows that its use is still limited in several educational processes being carried out in classrooms and schools. Particularly, in the field of educational guidance and despite the usefulness several authors award to ICT tools, the existing studies reveal their affordances are not being fully developed and its use is still scarce.

Based on this, a study to analyze the presence of Web 2.0 tools in educational guidance has been designed. A non experimental, quantitative research was carried out and the websites of the 155 Secondary Schools of the A Coruña province, Spain, were reviewed.

The results indicate than more than a half of the analyzed websites have a clear and friendly environment, which makes its use easier. However the availability of web 2.0 applications in the Department of Guidance websites is very small pointing out specially on the use of e-mail and blogs as the preferred tools. Technological training of counsellors should be improved as well as providing them with support and incentives are needed in order to properly integrate the ICT into their work.

Key Words: webtools 2.0, guidance departments, secondary education, educational guidance, information and communication technologies.

\section{Introducción}

El campo de la orientación educativa no ha permanecido indiferente ante las importantes transformaciones generadas a partir de la incorporación de las Tecnologías de la Información y de la Comunicación (TIC de ahora en adelante) al ámbito educativo. Como prueba de ello se pueden citar iniciativas como el proyecto "ICT Skills 2: herramientas TIC y formación para e-orientadores" financiado por la Comisión Europea dentro del programa Lifelong Learning (LLL, aprendizaje a lo largo de la vida 2007-2013). También cabe señalar otras acciones organizadas por esta misma institución, como la V Conferencia Europea sobre las TIC en la orientación, "Quality and Ethic in Web-Based Guidance", celebrada en junio del año 2011 en Goteborg (Suecia), en la que se puso de relieve el papel de las TIC en los servicios de orientación, valorándolas como recursos que facilitan nuevas perspectivas y oportunidades dirigidas a responder a necesidades cada vez más individualizadas y diversificadas. Más recientemente, lacob (2012) recoge ejemplos de buenas prácticas con TIC en el campo de la orientación. En definitiva tal y como afirma Cogoi (2002): "la utilización de las TIC es cada vez más necesaria y urgente para enfrentarse a los cambios de una sociedad sin fronteras" (p.5). 
Con el fin de valorar la integración de las TIC en el ámbito de la orientación, se ha realizado un estudio en torno a las características de las páginas web de los Departamentos de Orientación de los centros de educación secundaria de la provincia de A Coruña y se ha analizado en qué medida se están incorporando aplicaciones de la denominada Web 2.0. Como marco de referencia, y previamente a explicitar el diseño de la investigación, se abordará la doble perspectiva existente entre autores como Pantoja (2004) que considera que las TIC han dado lugar a un modelo propio denominado "modelo de orientación tecnológico" frente a otros expertos que postulan que si bien las TIC son valiosos recursos de apoyo para el orientador no pueden constituirse como un modelo (Vélaz de Medrano, 1998). Tras el análisis del doble planteamiento señalado en líneas anteriores, se procederá a clasificar y analizar las principales aplicaciones y herramientas web 2.0 susceptibles de uso por parte del orientador educativo. Finalmente, se describirá el método y el diseño de la investigación utilizado para, a continuación, presentar los resultados obtenidos.

\section{Antecedentes y fundamentación teórica}

Las TIC en la orientación educativa: ¿modelo tecnológico vs. recurso psicopedagógico?.

Los modelos en orientación son útiles porque, tal y como apuntan Grañeras y Parras (2009):

...configuran un marco de referencia para la investigación e intervención sin necesidad de recurrir directamente a las teorías más complejas en las que se enmarcan. Los modelos se sitúan, por así decirlo, entre la teoría y la práctica, a medio camino entre la abstracción y lo concreto. Sirven para interpretar y comprobar aquello que se intenta. (p.47).

No resulta sencillo realizar una clasificación de todos los modelos existentes en el ámbito de la orientación, dado que son diversas y numerosas las formulaciones existentes por parte de diferentes autores. No obstante, creemos que los seis modelos de intervención en orientación recopilados en la tabla 1 reflejan, no sólo aquellos comúnmente aceptados en el ámbito de la orientación, sino también a los autores más representativos que han trabajado sobre estas cuestiones.

TABLA 1. Diferentes clasificaciones de los modelos de intervención

\begin{tabular}{|l|l|l|l|l|l|l|}
\hline \multicolumn{1}{|c|}{ Autores } & Counseling & Consulta & Servicios & Programas & $\begin{array}{c}\text { Servicios } \\
\text { por } \\
\text { programas }\end{array}$ & Tecnológico \\
\hline Álvarez Rojo (1994) & & $\checkmark$ & $\checkmark$ & $\checkmark$ & & $\checkmark$ \\
\hline $\begin{array}{l}\text { Rodríguez Espinar, } \\
\text { Álvarez, Echeverría y } \\
\text { Marín (1993) }\end{array}$ & $\checkmark$ & $\checkmark$ & $\checkmark$ & $\checkmark$ & $\checkmark$ & $\checkmark$ \\
\hline $\begin{array}{l}\text { Bisquerra y Álvarez } \\
\text { (1998) }\end{array}$ & $\checkmark$ & $\checkmark$ & $\checkmark$ & $\checkmark$ & & \\
\hline Jiménez y Porras (1997) & $\checkmark$ & $\checkmark$ & & $\checkmark$ & \\
\hline $\begin{array}{l}\text { Vélaz de Medrano (1998) } \\
\text { Sampascual y otros }\end{array}$ & $\checkmark$ & $\checkmark$ & $\checkmark$ & $\checkmark$ & $\checkmark$ & $\checkmark$ \\
\hline $\begin{array}{l}\text { Sobrado y Ocampo } \\
\text { (1997) }\end{array}$ & $\checkmark$ & $\checkmark$ & $\checkmark$ & $\checkmark$ & $\checkmark$ & \\
\hline
\end{tabular}




\begin{tabular}{|l|l|l|l|l|l|l|}
\hline \multicolumn{1}{|c|}{ Autores } & Counseling & Consulta & Servicios & Programas & $\begin{array}{c}\text { Servicios } \\
\text { por } \\
\text { programas }\end{array}$ & Tecnológico \\
\hline Lázaro y Mudarra (2000) & $\checkmark$ & $\checkmark$ & $\checkmark$ & $\checkmark$ & $\checkmark$ & $\checkmark$ \\
\hline Repetto (2002) & $\checkmark$ & $\checkmark$ & $\checkmark$ & $\checkmark$ & & $\checkmark$ \\
\hline Pantoja (2004) & $\checkmark$ & $\checkmark$ & & $\checkmark$ & & $\checkmark$ \\
\hline Hervás Avilés (2006) & $\checkmark$ & $\checkmark$ & & $\checkmark$ & & \\
\hline
\end{tabular}

Fuente: Grañeras y Parras, 2009.

En contraposición con otros modelos más consolidados, se puede percibir cierta discrepancia entre los diferentes expertos en cuanto a la consideración del denominado "modelo tecnológico", ya que para algunos la tecnología está constituida por herramientas y recursos en los que se pueden apoyar el resto de modelos. Reconocen el modelo tecnológico, además de los autores señalados en la tabla 1, otros como Pantoja y Campoy (2001a) o Rodríguez Santero y Valverde (2003). Sin embargo, investigadores como Vélaz de Medrano (1998) se inclinan a valorar el uso de las TIC en la orientación como un recurso.

Desde un punto de vista funcional, para Pantoja (2004) el modelo tecnológico se basa en:

... la integración de las TIC en un programa comprensivo de intervención, dirigido preferentemente a la prevención y al desarrollo. Asimismo, este modelo despliega sus funciones alternando las dimensiones dentro-fuera del contexto en el que se lleve a cabo, directa-indirecta e individual. Según los casos, puede adoptar determinadas características de otros modelos de intervención (p. 189).

Este mismo autor resalta que para que el modelo tecnológico funcione debe estar adecuadamente integrado bajo un diseño organizativo holístico y dentro de una teoría que lo justifique y delimite. Igualmente, el modelo tecnológico debe responder a un modelo organizativo de una "e-escuela", es decir, un espacio en el que los orientadores y tutores digitales desarrollarían su labor. Se trata, según Pantoja y Zwierewicz (2008) de un nuevo entorno de y para el cambio, caracterizado por su flexibilidad, así como por su adaptabilidad a las características de cada sujeto y sin constricciones espacio-temporales, de materiales o personas.

En líneas generales, siguiendo a Pantoja (2004), se pueden identificar las siguientes características del modelo tecnológico:

- Sitúa la orientación en un escenario más amplio que el contexto donde se ubica, proyectándose en toda su extensión a diversos sectores: alumnos, familias, profesionales de la orientación y tutores.

- Facilita el intercambio de toda clase de información entre los implicados en el acto orientador.

- Confiere autonomía a los implicados.

- Permite un gran número de posibilidades de desarrollo de la acción orientadora.

- Se puede integrar en otros modelos y en el currículum de orientación (p.191).

A modo de síntesis, se sintetizan a continuación las principales ventajas e inconvenientes del modelo tecnológico de orientación (Pantoja, 2004).

En cuanto a las ventajas, cabe señalar las siguientes: El orientador se convierte en un consultor o asesor pudiendo realizar tareas de forma más flexible, adaptándose a los intereses y 
ritmo personales, así como posibilitando una intervención individual y/o grupal. En definitiva, se favorece la auto-orientación personal.

En relación a los inconvenientes, podrían citarse: La carestía de formación específica en TIC de los orientadores y el estado embrionario en el que todavía se encuentran las TIC en el ámbito de la orientación. De ahí que la función de asesoramiento on-line dista todavía de ser una auténtica realidad.

A diferencia de la concepción de las TIC que subyace al modelo tecnológico, ciertos autores como Vélaz de Medrano (1998), afirman que las TIC no tienen la entidad necesaria para ser consideradas como un modelo del mismo rango que el resto, por lo que deberían conceptualizarse simplemente como un recurso psicopedagógico muy valioso, el cual permite actuar como elemento vehicular o mediador de los aprendizajes y del desarrollo personal, académico y profesional de los destinatarios de la orientación.

Algunas de las razones que esta misma autora aduce para no considerarlo un modelo es que no se ajusta a ninguna de las acepciones que definen el concepto de modelo (como "representación de la realidad", como "muestra o ejemplo", como "prototipo" o "ideal a conseguir" en Orientación).

En lo que sí coinciden numerosos expertos es en el valor de las TIC, entendidas como recursos, en la práctica de la orientación. Así, Repetto et al. (1994) hablan de la plausible incorporación de estas herramientas tecnológicas a ámbitos como:

- Orientación personal: programas para la superación del estrés, prevenir la drogadicción, el desarrollo social y emocional o la toma de decisiones personales, entre otros.

- Orientación de la carrera: identificar conceptos clave del desarrollo de la carrera, especificar los datos relevantes de la formación ocupacional, preparar currículos, solicitudes o cartas de presentación, estructurar entrevistas profesionales, o desarrollar habilidades para la búsqueda de empleo y la eficacia en su ejercicio.

- Consejo académico: técnicas de estudio, elección de optativas y estudios futuros, etc.

- Diagnóstico: entrenamiento en la realización de test, administración, corrección e interpretación de pruebas.

- Administración: horarios, registros, estadísticas, etc.

- Formación de orientadores.

Por su parte, Lora (2009), concreta en tres grandes áreas el uso de las TIC en la orientación educativa, a saber: acción tutorial; atención a la diversidad y orientación académica y profesional. También Cabero (2003) argumenta que las TIC son especialmente útiles en la orientación para el desarrollo de "grandes actividades", como por ejemplo: la información escolar y profesional, la realización de acciones de tutorización; y también como herramientas para el diagnóstico y autodiagnóstico, la formación de los orientadores, y la gestión y la administración.

En definitiva, con independencia de que se considere o no el modelo tecnológico, lo relevante para el presente estudio es que, en ambos casos, se resalta la importancia de las TIC como elemento clave para llevar a cabo el proceso de orientación, ya que como indican Grañeras y Parras (2009): "por un lado facilita el trabajo de los profesionales de la orientación y agiliza la relación con orientados y, por otro lado, puede ser el marco global en el que se apoyan el resto de los modelos" (p.87). 


\section{Herramientas web 2.0 para la orientación educativa.}

Dentro del vasto campo de las TIC, la aparición de herramientas web 2.0 y de software social, está coadyuvando a potenciar nuevas formas de comunicación y posibilitando el desarrollo de un conocimiento compartido entre los usuarios y los profesionales de la educación. Así pues, la utilización de estas herramientas de marcado carácter dinámico ofrece al orientador la posibilidad de utilizar un lenguaje comprensible y constructivo, un discurso vinculado a situaciones y ejemplificaciones de la práctica profesional, un proceso dialógico de estructura horizontal, basado en la igualdad, en el dar y en el tomar (Domínguez, Álvarez y López Meneses, 2011). En suma, estas aplicaciones sociales, empleadas de modo adecuado, pueden generar verdaderas acciones de e-orientación, posibilitando el asesoramiento y la ayuda a distancia sin la obligación por parte del orientador ni del usuario de estar presente físicamente en horarios concretos en un lugar determinado (Offer, 1997; Tait, 1999).

Partiendo de estas consideraciones, ¿cuáles serían entonces las principales herramientas susceptibles de ser utilizadas en la orientación educativa?. Dada la amplia amalgama de aplicaciones basadas en el cloudcomputing, resultaría harto complicado poder ofrecer una respuesta unívoca a esta pregunta. No obstante, en un esfuerzo de síntesis se hará referencia a aquellas webtools que podrían considerarse de mayor utilidad para la acción orientadora en educación secundaria. Para ello, se propone una estructura basada en tres grandes categorías que, además de reseñarlas en la figura 1, se describirán de manera sucinta a continuación.

FIGURA 1. Clasificación de herramientas web 2.0 para la orientación educativa

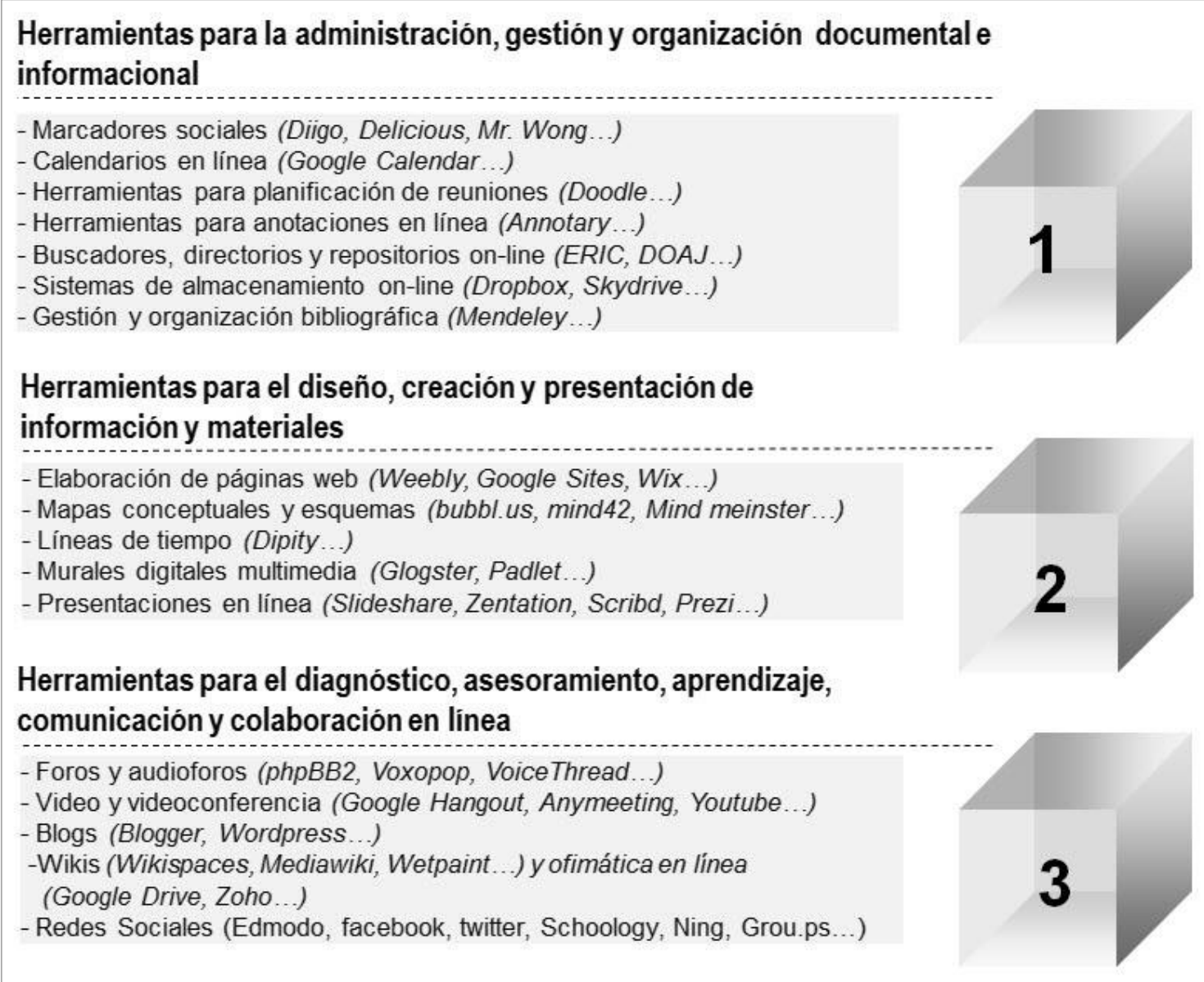

Fuente: elaboración propia 
Las "Herramientas para la administración, gestión y organización documental e informacional", son especialmente indicadas para que el orientador pueda utilizarlas como apoyo a cuestiones de índole administrativa, de gestión y de organización documental. También para categorizar y compartir eventos o documentación relevante con miembros de la comunidad educativa. Existe una amplia diversidad de aplicaciones de esta tipología, entre las que podrían destacarse:

- Los marcadores sociales: Permiten ordenar documentación y acceder a ella de forma online; crear o subscribirse a grupos de trabajo (e.g.: adherirse a grupos a los que pertenecen profesionales de la orientación; abrir grupos con temáticas concretas en las que pueda colaborar el orientado y el profesorado aportando información, como por ejemplo técnicas de estudio, convivencia, acciones tutoriales, etc.); valorar y etiquetar recursos relacionados con la orientación educativa; acceder y comentar recursos e información previamente valorada por otros orientadores o profesionales de la educación; asesorar al profesorado del centro en el uso de los marcadores sociales como herramienta para la contribuir a la mejora de procesos de aprendizaje significativo entre su alumnado, etc.

- Los calendarios en línea y las herramientas para la planificación de reuniones, como google calendar y doodle, respectivamente. Un orientador/a educativo podría tener digitalizada su agenda profesional, acceder a la misma desde cualquier dispositivo conectado a internet, planificar de forma autónoma o colaborativa (con otros orientadores o profesores) diversos proyectos para el centro (e.g. programa de acción tutorial; de promoción de la salud; convivencia, etc.). Este tipo de herramientas también serían muy adecuadas para que los orientadores asesorasen al alumnado de cara a fomentar su autonomía personal y mejorar su eficacia en lo referente a la organización de su tiempo de ocio y estudio, etc.

- Los directorios documentales, así como los repositorios en línea servirían para realizar consultas a la hora de elaborar adecuados diagnósticos psicopedagógicos; emplear la información recabada para la creación de programas educativos concretos; conocer casos similares a los que se producen en su centro educativo y buscar soluciones al mismo (e.g.: búsqueda de información para asesorar al profesor de educación física sobre adaptaciones curriculares a un alumno/a con parálisis cerebral), etc.

- Sistemas de almacenamiento on-line como Dropbox, Zyncro, SkyDrive y similares, que permiten al orientador digitalizar y guardar en la nube diferentes archivos de trabajo, pudiendo acceder a ellos cuando los necesite, en cualquier momento y lugar, así como compartirlos en el marco de proyectos colaborativos con otros profesionales de la orientación, profesorado, alumnado y/o familias.

- Los sistemas de gestión y organización bibliográfica como Mendeley o Zotero serían especialmente interesantes para la auto-formación del orientador, para compartir referencias y documentos con otros profesionales de la orientación, y para gestionar información de investigaciones y manuales que permitan una mejora en el quehacer diario.

En la categoría "Herramientas para el diseño, creación y presentación de información y de materiales", se englobarían aquellas aplicaciones que permiten al orientador diseñar, elaborar, crear y presentar información y/o materiales en formato hipermedia a fin de poder compartirlos con la comunidad educativa. Por ejemplo: herramientas para la elaboración de webs, mapas conceptuales, líneas de tiempo, murales digitales y presentaciones en línea. Todas ellas poseen grandes potencialidades, a continuación se comentan algunas de ellas:

- El orientador puede crear una web del Departamento de Orientación e integrar en ella diversas herramientas (blogs, foros, sistema de videoconferencia) a fin comunicarse con el profesorado, alumnado, familias. También puede elaborar materiales en línea sobre 
contenidos propios del ámbito de la orientación que sirvan de ayuda a docentes y estudiantes, por ejemplo, manuales sobre educación sexual, promoción de la salud, convivencia escolar, técnicas de estudio, atención a la diversidad, elaboración de WebQuest, orientación vocacional, etc. Otras utilidades serían las de publicar proyectos y acciones educativas desarrolladas en el propio centro educativo, portafolios de alumnos, elaboración de repositorio de contenidos, etc.

- Las herramientas que permiten elaborar mapas conceptuales y esquemas son especialmente adecuadas para, conjuntamente con el alumnado, organizar la información, desarrollar la capacidad de síntesis, trabajar el tratamiento de la información y la competencia digital, desarrollar capacidades metacognitivas, etc.

- Las líneas de tiempo como "dipity" son muy adecuadas para trabajar en materias relacionadas con Geografía e Historia y el orientador podría asesorar pedagógicamente sobre el uso de este tipo de recursos a los docentes. También es una webtool interesante para documentar la evolución de diversos programas o eventos realizados desde el Departamento de Orientación.

- Los murales digitales multimedia como "glogster" o "padlet" pueden ayudar a los alumnos con dificultades de aprendizaje, posibilitando el empleo de métodos de enseñanza basados en procesos constructivistas y en formas de trabajo colaborativo. Asimismo, en el alumno se favorecería el desarrollo de la creatividad y de la síntesis de contenidos (biografías, recensiones de libros, resúmenes de temas, viajes...). Igualmente, los murales digitales pueden servir como medio para la difusión de noticias, de determinados proyectos desarrollados por el Departamento de Orientación, elaboración de tormentas de ideas, etc.

- Las presentaciones en línea se configuran como herramientas con grandes potencialidades didácticas, ya que el orientador puede actuar como asesor del profesorado para que éstos realicen una correcta integración pedagógica de las mismas en sus clases o aprendan a utilizarlas como recursos para que el alumnado mejore sus habilidades comunicativas. El orientador también puede hacer uso de ellas para sus presentaciones a otros colectivos tanto del centro educativo (profesorado y estudiantes) como en reuniones con otros miembros de la comunidad educativa (familias) u otros agentes con los que puede trabajar.

En la categoría de "Herramientas para el diagnóstico, asesoramiento, aprendizaje, comunicación y colaboración en línea”, encontramos aplicaciones tanto asincrónicas (foros, audioforos, blogs, wikis, redes sociales...) como sincrónicas (sistemas de videoconferencia, chat integrado en wikis o redes sociales) a través de las cuales el orientador puede desarrollar acciones como las siguientes:

- En tareas de diagnóstico, los foros permiten, por ejemplo, participar o moderar grupos de discusión de expertos relacionados con el diagnóstico en educación (exposición de casos, elaboración de FAQ' s sobre aspectos concretos relacionados con el diagnóstico, compartir información sobre herramientas diagnósticas, investigación, solicitud de ayuda, etc.); así como disponer de un medio público de contacto con las familias de los alumnos para solucionar dudas y consultas.

Utilizando sistemas de videoconferencia, los orientadores podrían colaborar con otros colegas, consensuar diagnósticos en determinados casos y organizar sesiones individuales o grupales para desarrollar propuestas educacionales (temáticas desarrolladas en el plan de acción tutorial, etc.).

- En tareas de asesoramiento, mediante herramientas como los audioforos y los foros, sería posible realizar acciones de orientación individual o grupal con el alumnado, el profesorado 
y las familias; así como desarrollar una radio escolar y un podcast que sirva como un medio de asesoramiento en el que colaboren profesores y alumnos...

A través de sistemas de vídeo -como "google hangout" o similares-, se podrían organizar sesiones para plantear entrevistas de asesoramiento (individuales o en pequeño grupo) con expertos a distancia; asesorar al profesorado en la creación de videotutoriales y también sobre cómo involucrar al alumnado para desarrollar sistemas de aprendizaje constructivistas mediante estas herramientas de vídeo.

- En el ámbito informacional y comunicacional, herramientas como los blogs permiten: difundir materiales para tutorías, mostrar publicaciones y consejos sobre orientación profesional y vocacional, compartir experiencias de antiguos alumnos, así como incluir links, recursos y guías para el profesorado (Matas, 2011). También los wikis resultan de especial interés, sobre todo, para elaborar proyectos conjuntos con orientadores de otros centros educativos; participaren la edición colaborativa de documentos del centro (plan de acción tutorial, proyecto educativo, etc.), publicar recursos y materiales propios o de terceros, etc.

- Con el uso de redes sociales, el orientador podría crear grupos para desarrollar acciones formativas y de innovación; publicar noticias sobre temas relacionados con la orientación educativa (mediación escolar, atención a la diversidad, convivencia escolar, etc.); abrir canales de comunicación fluidos con familias, profesores y alumnos; asesorar al profesorado para utilizar las redes sociales en sus clases, etc. (Sobrado, Nogueira y García, 2013).

En definitiva, las herramientas web 2.0 se configuran como un aliado del orientador educativo que le permitirán desarrollar acciones en línea que superen las habituales restricciones de carácter espacial y temporal. Domínguez, Álvarez y López Meneses (2011) presentan ejemplos del uso de este tipo de aplicaciones en el ámbito de orientación en educación secundaria.

\section{Método}

El estudio realizado es de carácter cuantitativo, exploratorio y descriptivo. Se ha empleado un diseño no experimental de tipo transicional o transversal. No se han variado de forma intencional las variables independientes, de modo que como afirman Hernández Sampieri, Fernández Collado y Baptista (2007) "se observan los fenómenos tal y como se dan en su contexto natural, para después analizarlos" (p. 140). Siguiendo a Best (1982), se incluiría en los denominados "estudios de conjunto" que se caracterizan por su extensión y transversalita, así como por tratar un número de casos relativamente grande en un momento dado en donde se pueden obtener estadísticas deducidas de grupos de casos especiales.

Los datos se han recogido a finales del mes de mayo y principios de junio de 2013.

\section{Objetivos, hipótesis y preguntas de investigación}

Se identificaron las siguientes preguntas de investigación:

- ¿Desde el Departamento de Orientación de los centros educativos de la provincia de A Coruña se dispone de herramientas sincrónicas y/o asincrónicas para la acción orientadora? 
- ¿La webs/plataformas de los Departamentos de Orientación de los centros educativos de la provincia de A Coruña siguen criterios de usabilidad?

- ¿En qué medida se integran elementos multimedia en las webs/plataformas de los Departamentos de Orientación de los centros educativos de la provincia de A Coruña?

El objetivo general de la investigación ha sido identificar la presencia de aplicaciones web2.0 en los Departamentos de Orientación de Secundaria en la provincia de A Coruña, a través del análisis de sus páginas web/plataformas oficiales.

Más específicamente se pretendía:

- Conocer qué tipo de herramientas sincrónicas y asincrónicas están disponibles en los Departamentos de Orientación para llevar a cabo acciones informativas, de consultoría y asesoramiento a la comunidad educativa (estudiantes, profesorado y familias).

- Analizar aspectos técnico-funcionales de las webs/plataformas de los Departamentos de Orientación como la usabilidad y el grado de incorporación de elementos multimedia.

Además de los objetivos, se formuló la siguiente hipótesis: Existen diferencias significativas en cuanto a la disponibilidad de herramientas sincrónicas y asincrónicas en las páginas web de los Departamentos de Orientación en función de la tipología del centro (público, privado-concertado).

\section{Población objeto de estudio.}

El estudio se ha realizado sobre las webs/plataformas de los Departamentos de Orientación de todos los centros educativos de secundaria obligatoria de la provincia de A Coruña, públicos, privados y concertados. No se ha realizado ningún muestreo, sino que se analizaron la totalidad de las webs de los 155 centros educativos. Éstos se distribuyen en 95 centros públicos (lo que equivale a un $61,3 \%$ sobre el total), 54 concertados (que en términos porcentuales son el $34,8 \%$ ) y 6 privados (que suponen el 3,9\%). Para la identificación de todos los centros se han utilizado las bases de datos oficiales de la Consellería de Educación y Ordenación Universitaria de la Xunta de Galicia, accesibles desde https://www.edu.xunta.es/webcentros/.

\section{Procedimiento de recogida de información.}

Los datos se recogieron a partir del análisis documental de las webs/plataformas de los Departamentos de Orientación de los diversos centros de la provincia de A Coruña. Autores como Bisquerra (2009) o Del Rincón, Latorre y Sans (1995), destacan la versatilidad de esta técnica, así como su flexibilidad para examinar todo tipo de documentos y obtener información retrospectiva y referencial sobre determinadas situaciones o eventos.

Tras la consulta a las 155 páginas web de cada centro educativo se elaboró un protocolo de recogida de datos para cada una de las categorías de análisis que se explican en el siguiente párrafo. La codificación se realizó en una hoja de cálculo Excel de la siguiente forma: "sí" (en caso de presencia, codificado como "1") y "no" (en caso de ausencia, codificado como "2"). Hay que precisar que aquellos centros que carecían de web se codificaron como "0" (no consta). Tras la recopilación de todos los datos, éstos se importaron al programa SPSS versión 19 para realizar los diferentes análisis descriptivos y no paramétricos. 


\section{Categorías de análisis utilizadas.}

Seguidamente se describen las dos categorías empleadas para el análisis de las webs/plataformas de los Departamentos de Orientación (en adelante DO) de los diversos centros analizados.

- Categoría 1. Herramientas de comunicación sincrónicas y asincrónicas utilizadas para la consulta y el asesoramiento por parte del DO.

Esta categoría se analiza la presencia de las siguientes herramientas: Correo electrónico, mensajería interna y/o formularios electrónicos de contacto; Chat y mensajería instantánea; Redes sociales; Foros; Videoconferencia/Audioconferencia; Blogs y LMS (plataformas de gestión del aprendizaje).

Se han analizado estas aplicaciones por ser las más conocidas y extendidas en la actualidad. Para la selección de las mismas se han tenido en cuenta investigaciones previas como las desarrolladas por Nogueira-Pérez, Fernández-Sestelo, Muradás-López y Porta-Marín (2005); Pantoja y Campoy (2001b) y Sobrado $(2004,2006)$.

- Categoría 2. Aspectos técnico-funcionales de la web/plataforma utilizada por el DO.

Esta categoría se estructura en dos sub-categorías. Por una parte se pretende identificar la facilidad o no en cuanto al uso de las webs/plataformas analizadas (concretamente, si presentaban una adecuada organización de la información, estructurada en menús de navegación claros que permitiesen al usuario acceder de modo rápido e intuitivo a la información que fuese de su interés).Y, por otro, evaluar la existencia de un número suficiente de elementos multimedia (vídeos, presentaciones, animaciones, audio, materiales web, imágenes, realidad aumentada, etc.), teniendo en cuenta la amplitud de la propia web.

\section{Resultados}

De los 155 centros analizados, en 109 centros (70,3\%), el Departamento de Orientación disponía de un apartado propio en la web oficial del centro. Entre estos 109, había 25 que utilizaban herramientas externas (fundamentalmente blogs) como complemento a la web oficial de su centro. Un total de $44(28,4 \%)$ no disponían o no tenían espacio web, y tan sólo 2 centros $(1,3 \%)$ optaron por utilizar exclusivamente herramientas externas como blogger.

En primer lugar se efectuaron los análisis descriptivos y, posteriormente, para identificar si existían diferencias en cuanto a la tipología de centros, se realizó un análisis de contraste mediante Chi-cuadrado.

Como refleja el gráfico 1, en líneas generales, existe un escaso uso de las diferentes herramientas sincrónicas y asincrónicas por parte de los orientadores. Entre las aplicaciones más utilizadas se sitúan el correo electrónico/mensajería instantánea (15,5\%), seguido del blog (13,5\%) y de los LMS (7,1\%). El resto de las herramientas prácticamente no son utilizadas por los orientadores/as. 


\section{GRÁFICO 1. Herramientas de comunicación disponibles en los DO de secundaria de la provincia de A Coruña}

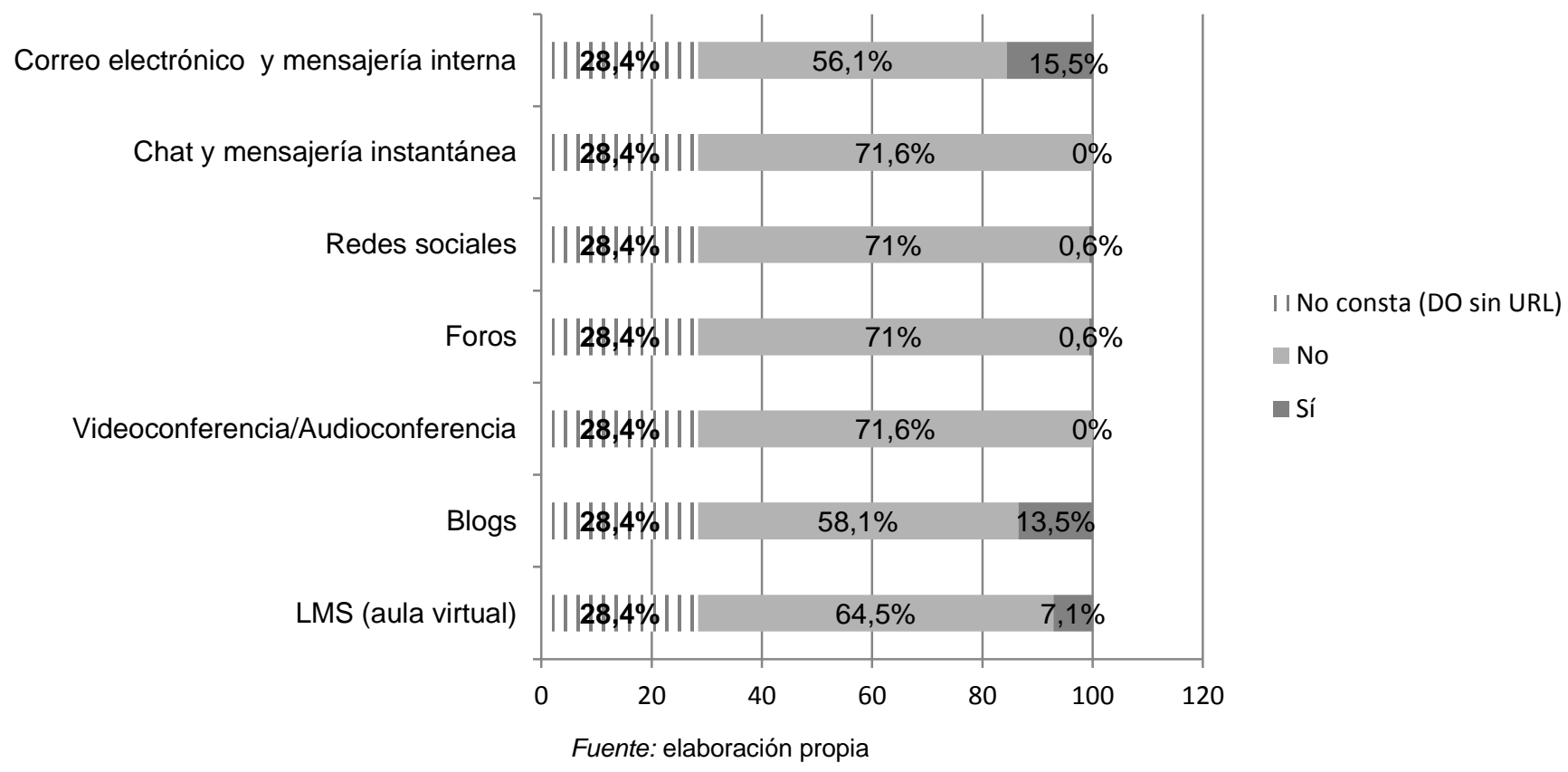

Estos resultados muestran un gran paralelismo con los obtenidos en la investigación desarrollada por Nogueira-Pérez, Fernández-Sestelo, Muradás-López y Porta-Marín (2005), cuyos participantes pertenecían a la red de orientadores escolares de los centros públicos y privados de la provincia de A Coruña, siendo un $40 \%$ orientadores del ámbito de secundaria. En el estudio señalado, los autores afirman que se pone de manifiesto la realidad actual de muchos centros en los cuales los orientadores no emplean las potencialidades de las TIC en su labor profesional. Y, por ejemplo, evidencian que éstos apenas usaban las herramientas TIC propuestas en su estudio (chat, web-forum y videoconferencia), siendo el correo electrónico la herramienta más utilizada (aunque advierten que con una frecuencia muy baja).

Por otra parte, en lo referido a aspectos como la usabilidad e incorporación de recursos multimedia (ver gráfico 2), se observa cómo la mayoría de páginas web cumplen criterios de usabilidad $(56,1 \%)$. Sin embargo, tan sólo un $11,6 \%$ incorporan algún tipo de recurso multimedia (vídeo, audio, animaciones, infografías, imágenes, etc.), lo cual supone un porcentaje escaso. 


\section{GRÁFICO 2. Inclusión de aspectos técnico-funcionales (usabilidad e incorporación de recursos multimedia) en las webs de los Departamentos de Orientación}

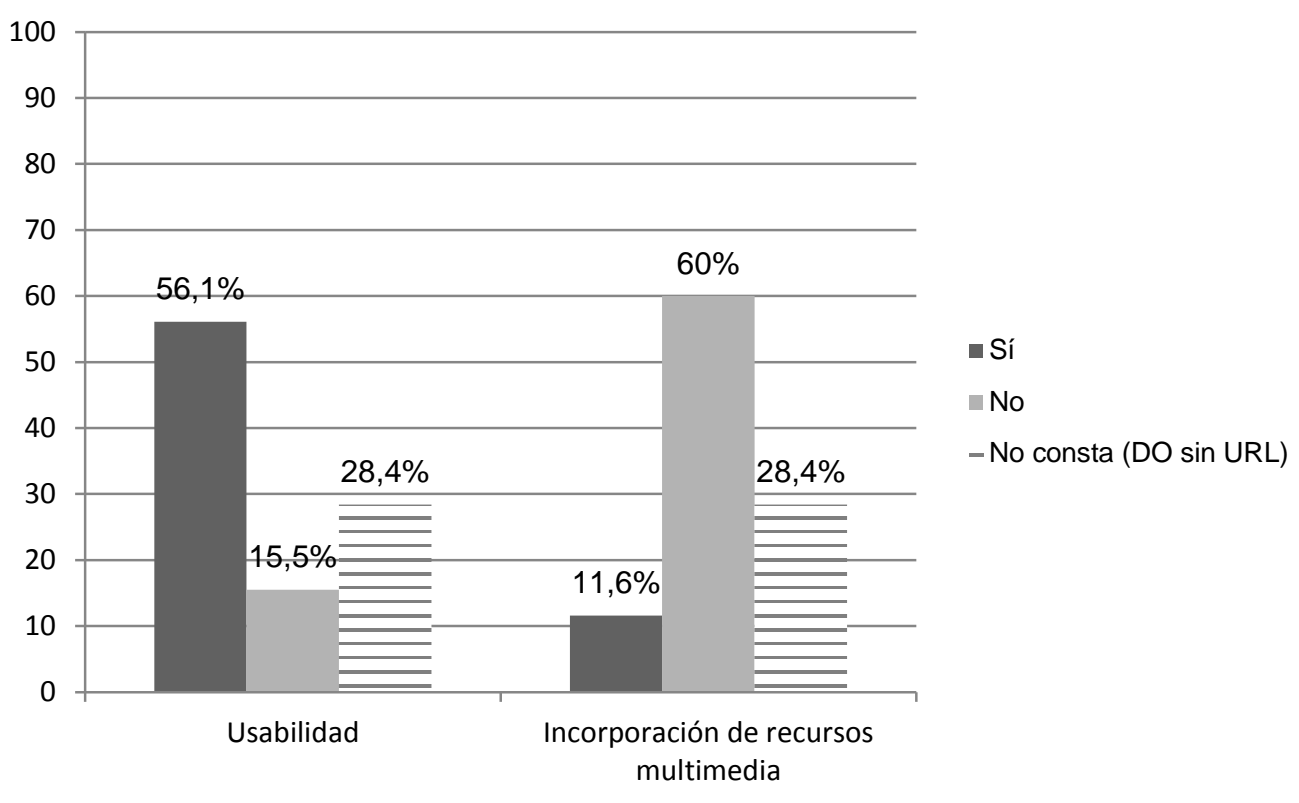

Fuente: elaboración propia

Tras realizar el análisis de los diversos estadísticos descriptivos para cada una de las categorías establecidas, de cara a confirmar o refutar la hipótesis planteada, se ha realizado una tabla de contingencia de 2x2 y se aplicó el estadístico de contraste Chi-cuadrado para variables categóricas.

En los análisis, únicamente se han considerado los centros que disponían de web propia del Departamento de Orientación. Además, se ha dicotomizado la variable nominal "tipo de centro", de forma que se ha unido en una categoría los centros concertados y privados, ya que estos últimos tenían frecuencias de cero en la mayoría de los casos.

Solamente se han encontrado diferencias significativas según la tipología de centro (público y concertado/privado) en la herramienta "correo electrónico y mensajería interna" (tabla 2). 
TABLA 2. Prueba Chi-cuadrado para la variable "correo electrónico y mensajería interna *tipo de centro"

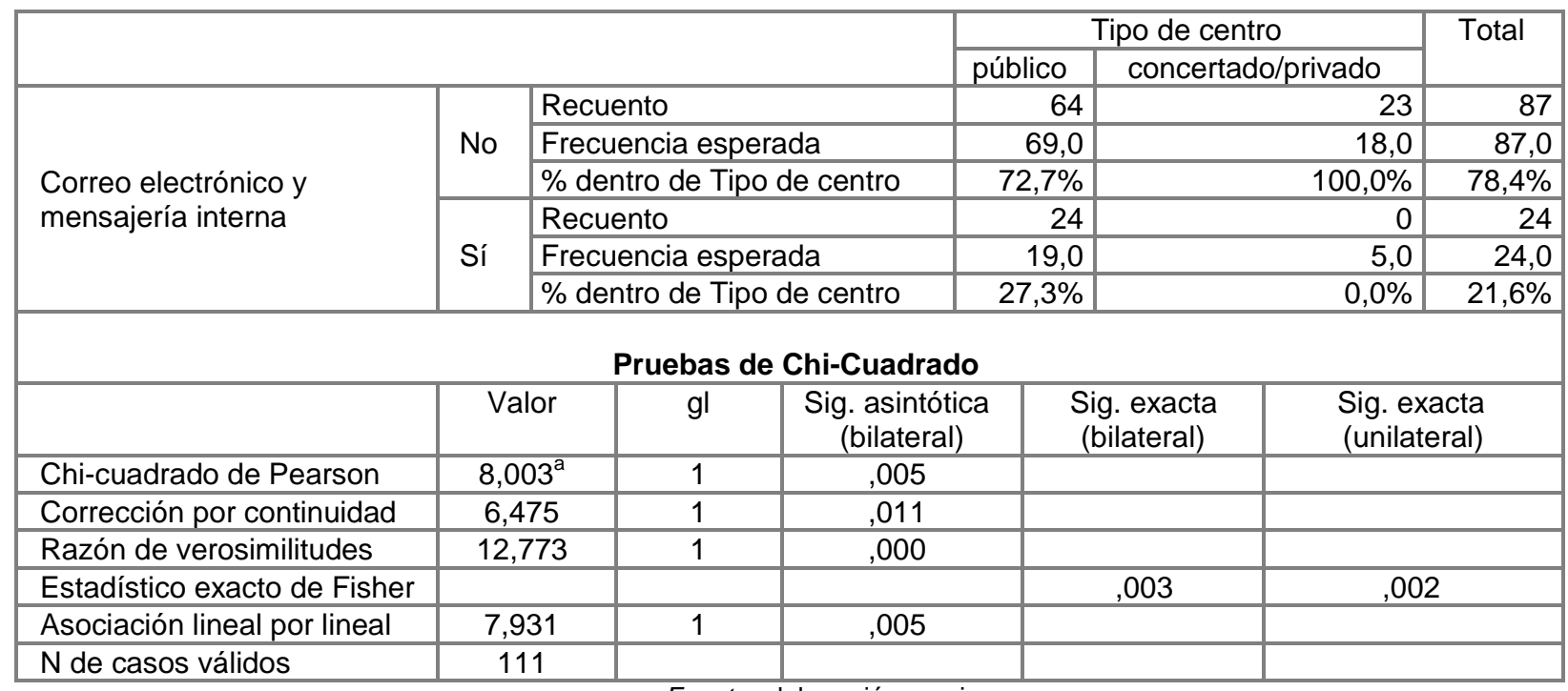

Fuente: elaboración propia

Como se puede observar en la tabla precedente, y dado que existe una casilla con una frecuencia esperada inferior a 5, la significación puede interpretarse en base a la corrección por continuidad de Yates y a la razón de verosimilitudes.

Aunque es mayor cantidad de centros públicos que de privados-concertados, se puede apreciar que la disponibilidad del correo electrónico entre los centros analizados presenta diferencias significativas (razón de verosimilitudes con $p$-valor=,000).

En ambas tipologías de centro, la presencia del correo electrónico es limitada, si bien, es mayor en centros públicos (un $27,3 \%$ de los encuestados), que en privados-concentrados, en cuyo caso es nulo.

\section{Conclusiones}

Los resultados obtenidos nos ofrecen una visión global del tipo de herramientas on-line que se pueden encontrar en las webs de los Departamentos de Orientación de educación secundaria y del grado de incorporación de recursos multimedia y su usabilidad.

A modo de síntesis, las principales conclusiones del estudio exploratorio que se ha realizado serían las siguientes:

- La disponibilidad de diferentes herramientas de comunicación para el asesoramiento on-line es muy limitada. Dentro de estos bajos niveles destaca el correo electrónico y los blogs como principales webtools.

- En un 7,1\% de centros se han encontrado cursos, implementados a través de plataformas de teleformación, relacionados con el ámbito de la orientación educativa.

- Más de la mitad de las webs analizadas $(56,1 \%)$ poseen un entorno claro y amigable que permite la facilidad de uso por parte del usuario.

- Existe un amplio porcentaje de páginas web/plataformas (60\%) que no incorporan elementos multimedia entre sus recursos: como vídeos, presentaciones, animaciones, etc. 
En el apartado siguiente se ofrecerán algunas reflexiones a partir de las cuales se quiere promover una visión más global y holística de la investigación realizada. Apoyándose en otros estudios, se pretende explicar y comprender el panorama que se desprende de los datos encontrados $y$, desde esta perspectiva, vislumbrar ciertas tendencias hacia las que habría que enfocar los esfuerzos y concretar las acciones para una adecuada integración de las TIC en los procesos de orientación educativa

\section{Discusión y prospectiva}

Los resultados obtenidos dibujan un contexto poco favorable respecto a la integración de las TIC en las tareas de orientación y, particularmente, ponen de manifiesto que no se están aprovechando adecuadamente las potencialidades de las herramientas vinculadas a la web 2.0. Esta situación no es diferente a la señalada en otros estudios más generales en los que se concluye que, por diversos factores tanto de carácter interno como externo, y a pesar del considerable incremento en equipamiento tecnológico, ni se ha generalizado ni extendido el uso de la tecnología ni, sobre todo, su incorporación está generando los frutos esperados en cuanto a la mejora sustancial de los procesos educativos en las instituciones escolares (Davis, Preston y Sahin, 2009; Law, Pelgrum y Plomp, 2008; Sigalés, Mominó, Meneses y Badía, 2008; Tondeur, Valcke y Van Braak, 2008; Fundación Telefónica, 2011). El ámbito de la orientación no es ajeno a esta tendencia detectada tanto a nivel nacional como internacional, y parece que los esfuerzos desarrollados por algunos autores en la línea de destacar tanto la necesidad como las ventajas de integrar las TIC en las acciones de orientación no han tenido el eco suficiente (Cabeza, 2010; Campoy y Pantoja, 2003; Cogoi, 2002, 2005; lacob, 2012; Lora, 2009; Pantoja, 2004; Pantoja y Campoy, 2001 a y b; Pantoja y Zwierewicz, 2008; Repetto y Malik, 1998; Rodríguez Santero y Valverde, 2003; Sobrado, 2004, 2006).

Las razones que pueden explicar este escaso desarrollo de lo que algunos autores denominan e-orientación (Campoy y Pantoja, 2003) o, cuando menos, la reducida utilización de las herramientas TIC en el campo de la intervención orientadora, son de muy diverso origen. Hay que tener en cuenta que ni en décadas precedentes las tecnologías informáticas aplicadas a la orientación tuvieron gran aceptación ni ahora se dispone de suficiente y diverso software específico que haya sido experimentado y validado. En este sentido, son poco frecuentes propuestas como las que refieren algunos autores que dan cuenta de la creación y utilización de determinadas herramientas (Cuevas, 2011; Lozano-Martínez y Alcaraz-García, 2011; Sanz, Gil y Marzal, 2007) o que analizan el uso de internet en una de las líneas de intervención de la orientación (Reile y Harris-Bowlsbey, 2000). En este sentido, conviene ser conscientes de las limitaciones de los propios centros educativos en cuanto a la disponibilidad de recursos específicos para las labores orientadoras y las dificultades por parte de los profesionales de la orientación para crear sus propios materiales e incluso acceder a herramientas que tendrían que adaptar a su contexto. La soledad del orientador en los centros y, a veces, la falta de apoyo para colaborar con otros colegas y/o docentes, puede revertir negativamente en el desarrollo de proyectos vehiculados a través de la tecnología.

$Y$ junto a factores contextuales, como los que se acaban de citar, hay que considerar la posición del propio orientador respecto a la tecnología que, en gran medida va a depender de la formación que posee, sus actitudes y sus experiencias previas. El factor personal ha sido reconocido como fundamental en diversos estudios que analizan la integración de las TIC en la educación (Ertmer y Ottenbreif-Leftwich, 2010;) y, concretamente, la necesidad de cubrir tanto la alfabetización como la capacitación tecnológica se han revelado claves para posibilitar un uso adecuado de la tecnología en la enseñanza. En el ámbito de la orientación educativa se han 
realizado diversos estudios sobre las destrezas, habilidades y competencias de los orientadores. A nivel internacional podemos citar los trabajos de McCarthy (2004) y Pelling (2009) y en el ámbito español, los realizados por Sobrado y sus colaboradores para identificar las competencias de los orientadores (Sobrado, Ceinos y Fernández, 2010; Sobrado, Fernández, Ceinos y García, 2010). El análisis de las competencias del orientador sería un buen punto de partida para estudiar los perfiles profesionales y, desde ahí, establecer acciones formativas, al menos, en un doble sentido: a) que permitiesen que cada profesional pudiese construir su propio itinerario de aprendizaje y desarrollo profesional, y b) que las instituciones responsables de la formación en ejercicio, pudieran organizar sus propuestas formativas para ir cubriendo los diversos ámbitos de competencias y dar respuesta a los distintos niveles de dominio que se puedan contemplar.

La integración de las TIC en el ámbito educativo en general, y en la orientación en particular, no es únicamente una cuestión técnica que pueda resolverse a través de medidas de intervención como las de índole formativa que citamos anteriormente. Es necesario atender también los aspectos actitudinales (Vinluan, 2011) y, tener en cuenta la necesidad de que los profesionales de la orientación desarrollen percepciones positivas y una disposición favorable.

Pero además, la incorporación de las TIC no está exenta de aspectos éticos (Mallen, Vogel y Rochlen, 2005; McCrickard y Butler, 2005; Valverde y Fernández 2013)y, por tanto, estos deben estar presentes en los análisis que se realicen sobre esta temática y hay que tomar conciencia de sus repercusiones cuando se intente ofrecer la tecnología como herramienta necesaria a los profesionales de la orientación. Evitar suspicacias como las relativas al cuestionamiento de su labor cuando se recomienda introducir las TIC o incluso disipar el miedo de algunos a que la tecnología pueda sustituirles en su labores de orientación, son aspectos que no deben obviarse (Sobrado, 2006).

Dar a voz a los propios orientadores para conocer sus perspectivas, analizar sus referentes formativos y sus trayectorias personales y profesionales, resulta imprescindible para completar el panorama que se ofrece a partir del estudio que estamos presentando y, sobre todo, de cara a proponer medidas dirigidas a incrementar tanto cuantitativa como cualitativamente el uso de las TIC en la orientación educativa. La realización de indagaciones en las que analiza la perspectiva del orientador -como el desarrollado Pantoja y Campoy (2001b) o Nogueira-Pérez, FernándezSestelo, Muradás-López, y Porta-Marín (2005) - u otros más específicos como el realizado por Ocampo, Caeiro y Sarmiento (2012) sobre la utilización de las TIC en la función tutorial en educación infantil-, son ejemplos que pueden seguirse y extenderse a otros niveles educativos y/o regiones.

En definitiva, como han apuntado Pantoja y Zwierewicz (2008) para que las TIC se conviertan en una herramienta cotidiana en la orientación educativa son necesarios una serie de cambios de tipo material (en cuanto a infraestructuras y materiales), formativo (mejorando la capacitación de orientadores y tutores), metodológico (fomentar acciones y estructuras que requieran el uso de las TIC en las aulas y los centros) y conceptual (construir y desarrollar una cultura institucional y profesional en la cual las TIC constituyan herramientas cotidianas en las acciones de orientación).

\section{Referencias bibliográficas}

Álvarez Rojo, V. (1994). Orientación educativa y acción orientadora. Relaciones entre la teoría y la práctica. Madrid: EOS.

Best, J.W. (1982). Cómo investigar en educación. Madrid: Ediciones Morata. 
Bisquerra, R. (2009). Metodología de la investigación educativa. Madrid: La Muralla.

Bisquerra, R. y Álvarez González, M. (1998). Concepto de orientación e intervención psicopedagógica. En R. Bisquerra, (Coord.). Modelos de orientación e intervención psicopedagógica (pp. 9-22). Barcelona: Praxis.

Cabero, J. (2003). Las nuevas tecnologías aplicadas a los procesos de orientación educativa. En B. Bermejo y J. Rodríguez (Dirs.). La orientación educativa y la acción tutorial en enseñanza secundaria (pp. 1-23). Sevilla: GID.

Cabeza, A. (2010). Importancia de las tecnologías de la información y la comunicación en la orientación educativa. Pedagogía Magna, 8, 26-33.

Campoy, T. J. y Pantoja, A. (2003). Propuestas de «e-orientación» para una educación intercultural. Comunicar, 20, 37-43.

Cogoi, C. (2002). Videoconferencia y orientación. Ámbitos de aplicación y ejemplos de buenas prácticas. Revista Española de Orientación y Psicopedagogía, 13(1), 5-15.

Cogoi, C. (2005). ICT Skills for Guidance Counsellors. Bolonia: Outline Edizione.

Davis, N., Preston, C. y Sahin, I. (2009). ICT teacher training: Evidence for multinivel evaluation from a national initiative. British Journal of Educational Technology, 40(1), 135-48.

Del Rincón, D., Latorre, A. y Sans, A. (1995).Técnicas de investigación en ciencias sociales. Madrid: Dykinson.

Domínguez, G., Álvarez F. J. y López Meneses, E. (2011). Orientación educativa y tecnologías de la información y la comunicación. Nuevas respuestas para nuevas realidades. Alcalá de Guadaíra (Sevilla): Editorial MAD.

Ertmer, P. A. y Ottenbreit-Leftwich, A.T. (2010). Teacher Tecnology Change; How Knowledge, Confidence, Beliefs and Culture Intersect. Journal of Research on Technology in Education, 42(3), 255-284.

Fundación Telefónica (2011). Las TIC en educación. Realidad y expectativas. Madrid: Editorial Ariel y Fundación Telefónica.

Grañeras, M. y Parras, A. (Coords.). (2009). Orientación educativa: fundamentos teóricos, modelos institucionales y nuevas perspectivas. Madrid: Edita Centro de Investigación y Documentación Educativa (CIDE).

Hernández Sampieri, R., Fernández Collado, C. y Baptista, P. (2007). Fundamentos de metodología de la investigación. México: McGraw-Hill.

Hervás Avilés, M. R. (2006). Orientación e intervención psicopedagógica y procesos de cambio. Granada: Grupo Editorial Universitario.

lacob, M. (2012).Good practices in the use of ICT in providing guidance and counseling. Bucarest: Institute of Educational Sciences.

Law, N.,Pelgrum, W.J. y Plomp, T. (Eds.) (2008). Pedagogy and ICT use in schools around the world: Findings from the IEA SITES 2006 study. Hong Kong: CERC-Springer.

Jiménez, R. y Porras, R. (1997). Modelos de acción psicopedagógica: entre el deseo y la realidad. Málaga: Aljibe.

Lázaro, A. y Mudarra, M. J. (2000). Análisis de los estilos de orientación en equipos psicopedagógicos. Contextos Educativos, 3, 253-280.

Lora, J. A. (2009). Las TICs en orientación. Innovación y experiencias educativas, 19, 1-8. 
Lozano-Martínez, J. y Alcaraz-García, S. (2011). Software educativo para la enseñanza de competencias emocionales en alumnado con trastornos del espectro autista. Educación XX1, 14(2), 189-212.

Mallen, M. J., Vogel, D. L. y Rochlen, A. B. (2005). The practical aspects of online counseling: ethics, training, technology, and competency. The Counseling Psychologist, 33, 776-818.

Matas, A. (2011). Los blogs como recurso para la orientación. En M.A. Fernández Jiménez,E. Mena Rodríguez (Coords.), Tutor 2.0: aplicaciones para entornos virtuales de aprendizaje(115-128). Málaga: Aljibe.

McCarthy, J. (2004). The skills, training and qualifications of guidance workers. International Journal for Educational and Vocational Guidance, 4, 159-178.

McCrickard, M. P. y Butler, L. T. (2005). Cybercounseling: A new modality for counselor training and practice. International Journal for the Advancement of Counselling, 27, 101-110.

Nogueira-Pérez, M. A., Fernández-Sestelo, M. M., Muradás-López, M. y Porta-Marín, M. I. (2005).El uso de las TIC para la orientación educativa a distancia: Valoraciones de los orientadores de A Coruña. VIII Congreso Galego-Portugués de Psicopedagoxía, Braga, 14-16 de septiembre.

Offer, M. (1997). A review of the use of computer-assisted guidance and the Internet in Europe. Dublin: National Centre for Guidance in Education.

Pantoja, A. (2004). La intervención psicopedagógica en la sociedad de la información. Madrid: EOS.

Pantoja, A. y Campoy, T.J. (2001a). Un modelo tecnológico de orientación universitaria. En L.M. Villar Angulo (Coord.), La universidad, evaluación educativa e innovación curricular (pp.95128). Sevilla: ICE de la Universidad deSevilla.

Pantoja, A. y Campoy, T.J. (2001b). El orientador ante las nuevas tecnologías. En @gora Digital, 2, pp. 1-12.

Pantoja, A. y Zwierewicz, M. (2008). Procesos de orientación en entornos virtuales de aprendizaje. Revista Española de Orientación y Psicopedagogía, 19(3), 282-290.

Pelling, N. (2009). The use of email and the Internet in counselling and psychological service: what practitioners need to know. Counselling, Psychotherapy, and Health, 5, 1-25.

Reile, D. M. y Harris-Bowlsbey, J. (2000). Using the Internet in career planning and assessment. Journal of Career Assessment, 8, 69-84.

Repetto, E. et al. (1994).Orientación educativa e intervención psicopedagógica. Madrid: UNED.

Repetto, E. (Dir.). (2002). Modelos de orientación e intervención psicopedagógica. Madrid: UNED.

Repetto, E. y Malik, B. (1998). Nuevas tecnologías aplicadas a la orientación. En Bisquerra, R. (Coord.), Modelos de orientación e intervención psicopedagógica (pp. 363-374). Barcelona: Praxis.

Rodríguez Espinar, S., Álvarez, M., Echeverría, B. y Marín. A. (1993). Teoría y práctica de la orientación educativa. Barcelona: PPU.

Rodríguez Santero, J. y Valverde, A. (2003). Tecnologías al servicio de la orientación y de apoyo al aprendizaje. Comunicar, 10(20), 89-95.

Sampascual, G., Navas, L. y Castejón, J.L. (1999). Funciones del orientador en primaria y secundaria. Madrid: Alianza. 
Sanz, J., Gil, J. M. y Marzal, A. (2007). HIA. Herramienta informática para el asesoramiento, Revista de Investigación Educativa, 25(2), 305-326.

Sobrado, L. (2004). Utilización das Tecnoloxías da Información e da Comunicación polos profesionais da orientación. Revista Galega do Ensino, 43, 89-106.

Sobrado, L. (2006). Las competencias de los orientadores en el ámbito de las TIC (Tecnologías de la Información y la Comunicación): diagnóstico y desarrollo. Estudios Sobre Educación, 11, 27-43.

Sobrado, L., Ceinos, M.C. y Fernández, E. (2010). Planificación y desarrollo de un mapa de habilidades TIC en Orientación. Comunicar, 35(18), 167-174, doi:10.3916/C35-2010-03-10

Sobrado, L., Fernández, E., Ceinos, M.C. y García, R. (2010). Rol de las TIC en la e-formación y orientación a lo largo de la vida:análisis de la realidad europea. Revista de Orientación y Psicopedagogía, 21(2), 271-282.

Sobrado, L. y Ocampo, C. (1997). Evaluación psicopedagógica y Orientación Educativa. Barcelona: Estel.

Tait, A. (1999). Face-to-face and at a distance: The mediation of guidance and counselling through the new technologies. British Journal of Guidance and Counsellor, 27(1), 113-122.

Tondeur, J., Valcke, M. y Van Braak, J. (2008). A multidimensional approach to determinants of computer use in primary education: Teacher and school characteristics. Journal of Computer Assisted Learning, 6(24), 494-506.

Valverde, J. y Fernández, M. R. (2013). El bienestar subjetivo ante las buenas prácticas educativas con TIC: Su influencia en el profesorado innovador. Educación XX1, 16(1), 255280.

Vélaz de Medrano, C. (1998). Orientación e intervención psicopedagógica. Concepto, modelos, programas y evaluación. Málaga: Aljibe.

Vinluan, L.R. (2011). The Use of ICT in School Guidance: Attitudes and Practices of Guidance Counselors in Metro Manila, the Philippines. International Journal for the Advancement of Counselling, 33(1), 22-36.

\section{Fuentes electrónicas}

Cuevas, V.J. (2011). Orientación 2.0. En J. Hernández Ortega M. Pennesi, D. Sobrino, A. Vázquez, Experiencias educativas en las aulas del siglo XXI. Innovación con TIC (286-290). Barcelona: Editorial Ariel. Recuperado el 26 de Enero2014: http://www.ciberespiral.org/attachments/225_Experiencias_educativas20.pdf

Ocampo, C. I., Caeiro, M. y Sarmiento, J.A. (2012). Las TIC y la función tutorial en la etapa de educación infantil: La opinión del profesorado de centros públicos del sur de Galicia. REOP, 23(2), 60-78. Recuperado el 5 de Junio de 2013 de: http://www.uned.es/reop/pdfs/2012/232\%20-\%200campo.pdf

Sigalés, C., Mominó, J., Meneses, J. y Badía, A. (2008). La integración de internet en la educación escolar española: situación actual y perspectivas de futuro. Recuperado el 5 de Junio de 2013 de:

http://www.uoc.edu/in3/integracion_internet_educacion_escolar/esp/informe.html 
Sobrado, L., Nogueira, M.A. y García, R. (2013). Las redes sociales de internet en el ámbito de la orientación educativa y profesional. XVI Congreso AIDIPE. Investigación e Innovación Educativa al Servicio de Instituciones y Comunidades Globales, Plurales y Diversas, Alicante, 4-6 septiembre. Recuperado el 26 de Enero de 2014 de:

http://www.uv.es/aidipe/congresos/Actas XVI Congreso.pdf

Fecha de entrada: 26 de octubre 2013 Fecha de revisión: 3de diciembre 2013 Fecha de aceptación: 26 de abril 2014 